PHYSICAL REVIEW D 94, 024004 (2016)

\title{
Compact and extended objects from self-interacting phantom fields
}

\author{
Vladimir Dzhunushaliev" \\ Department Theoretical and Nuclear Physics, KazNU, Almaty 050040, Kazakhstan \\ and IETP, Al-Farabi KazNU, Almaty 050040, Kazakhstan \\ Vladimir Folomeev ${ }^{\dagger}$ \\ Institute of Physicotechnical Problems and Material Science of the NAS of the Kyrgyz Republic, \\ 265 a, Chui Street, Bishkek 720071, Kyrgyz Republic \\ Arislan Makhmudov ${ }^{\ddagger}$ \\ Institute of Gravitation and Cosmology, Peoples Friendship University of Russia, \\ 6 Miklukho-Maklaya Street, Moscow 117198, Russia \\ Ainur Urazalina ${ }^{\S}$ \\ Department of Physics, California State University Fresno, Fresno, California 93740-8031, USA \\ and Department Theoretical and Nuclear Physics, KazNU, Almaty 050040, Kazakhstan \\ Douglas Singleton \\ Department of Physics, California State University Fresno, Fresno, California 93740-8031, USA \\ and ICTP South American Institute for Fundamental Research, UNESP, \\ Universidade Estadual Paulista Rua Dr. Bento T. Ferraz 271, 01140-070 São Paulo, São Paulo, Brazil \\ John Scott \\ Department of Physics, California State University Fresno, Fresno, California 93740-8031, USA \\ (Received 12 May 2016; published 5 July 2016)

\begin{abstract}
In this work, we investigate localized and extended objects for gravitating, self-interacting phantom fields. The phantom fields come from two scalar fields with a "wrong-sign" (negative) kinetic energy term in the Lagrangian. This study covers several solutions supported by these phantom fields: phantom balls, traversable wormholes, phantom cosmic strings, and "phantom" domain walls. These four systems are solved numerically, and we try to draw out general, interesting features in each case.
\end{abstract}

DOI: $10.1103 /$ PhysRevD.94.024004

\section{INTRODUCTION}

The current astronomical and cosmological observations indicate that the Universe is in an epoch of accelerated expansion. The source of this acceleration, dubbed dark energy (DE), is now under active investigation (for a review, see the book [1] and references therein). One of the distinctive properties of $\mathrm{DE}$ is its large negative pressure, which is comparable in magnitude to its energy density. To model DE, various approaches are employed, from the simplest $\Lambda \mathrm{CDM}$ model, where the DE energy density comes from a cosmological constant which does not change during the evolution of the Universe, to modified and multidimensional theories of gravity.

\footnotetext{
v.dzhunushaliev@gmail.com

vfolomeev@mail.ru

*arslan.biz@gmail.com

§Y.A.A.707@mail.ru

dougs@csufresno.edu

ๆjohnmlscott@mail.fresnostate.edu
}

One of the most popular ways to describe DE is through models with various scalar fields. For such fields, one can introduce some effective equation of state $p=w \varepsilon$, relating the pressure of the fields (i.e., $p$ ) with the energy density of the fields (i.e., $\varepsilon$ ). It then becomes possible to subdivide all such fields into two large classes: nonphantom fields, for which $w>-1$ (for instance, quintessence scalar fields), and phantom fields, for which the equation-of-state parameter $w$ is less than -1 . The recent observational estimates give $w=-1.10 \pm 0.14(1 \sigma)[2]$ and $w=-1.069_{-0.092}^{+0.091}$ [3]; i.e., the possibility is not excluded that some of the matter filling the Universe acts as phantom matter.

When describing the accelerated expansion of the Universe, it is usually assumed that DE is distributed homogeneously and isotropically on the largest scales. This, however, does not exclude a possibility that DE may cluster on relatively small scales comparable to sizes of galaxies or even of stars where one might expect an interesting interplay between the normal tendency of gravity to pull matter together versus the tendency of $\mathrm{DE}$ to act repulsively. For these cases, one could form 
compact objects, dubbed DE stars, both with a trivial and with a nontrivial topology of the associated spacetime [4]. In particular, if these types of objects contain phantom scalar fields one can construct localized solutions that can be used in describing configurations both with a trivial (starlike) [5] and with a nontrivial (wormholelike) spacetime topologies [6].

Apart from the aforementioned star- and wormholelike spherically symmetric configurations, one can consider extended objects supported by scalar fields but having symmetries other than spherical. One can look for cylindrically symmetric cosmic strings. Cosmic strings are extended configurations which could form in the early Universe at phase transitions associated with spontaneous symmetry breaking [7]. Different types of cosmic strings have been considered in the literature (see, e.g., Ref. [8]). Of particular interest to the phantom cosmic strings found in this paper are cosmic strings constructed from various types of scalar fields $[9,10]$, including models with two interacting scalar fields [11].

Another category of extended objects are planar symmetric domain walls which are topological defects arising in both particle physics and cosmology (see, e.g., Refs. [8,12] and references therein). They separate a spacetime into several domains along a single coordinate. The domain walls we find here arise from regions with fast spatial variation of the scalar fields. Depending on the behavior of the field near the wall, solutions describing such objects can be subdivided into thin-wall solutions (see, e.g., [13]), for which the scalar-field energy density can be replaced by the delta function, and thick-wall solutions [14].

It is clear that the characteristics of the aforementioned compact and extended objects will depend on the specific type of fields employed in their modeling. In the present paper, we consider all four types of configurations (domain walls, boson "stars", wormholes, cosmic strings) constructed from two interacting phantom scalar fields. One caveat in regards to the "star" solutions of the present work is that they have masses on the order of the Planck massthey are not objects with a stellar mass. For this reason, the spherically symmetric solutions that we find in this work are called phantom balls. The systems with two ordinary scalar fields are well known from quantum field theory, where they are used to obtain solitary wave solutions [15]. When a gravitational field is present, such systems have also been repeatedly considered in the cosmological and astrophysical contexts (see Ref. [16]). In our previous works, we have obtained a number of solutions with two scalar fields (both normal scalar fields and phantom scalar fields) which can be employed to describe astrophysical objects and also when considering cosmological solutions. We have considered regular spherically and cylindrically symmetric solutions [17-19], cosmological solutions $[20,21]$, and thick brane solutions supported by normal and ghost scalar fields [22]. Here we will extend those studies.

\section{GENERAL EQUATIONS}

In the simplest case, a phantom scalar field can be introduced by changing the sign of the kinetic term in the Lagrangian. Consistent with this, we choose the Lagrangian for two scalar fields $\phi, \chi$ as in Ref. [18],

$L=-\frac{R}{16 \pi G}-\left[\frac{1}{2} \partial_{\mu} \phi \partial^{\mu} \phi+\frac{1}{2} \partial_{\mu} \chi \partial^{\mu} \chi-V(\phi, \chi)\right]$,

where $R$ is the scalar curvature, $G$ is the Newtonian gravitational constant. We have designated the two scalar fields in (1) as phantom fields due to fact that we have taken their kinetic terms (i.e., $-\frac{1}{2} \partial_{\mu} \phi \partial^{\mu} \phi$ and $-\frac{1}{2} \partial_{\mu} \chi \partial^{\mu} \chi$ ) to have the "wrong" sign (i.e., a -sign) rather than a the "correct" sign (i.e., a +sign). More precisely, though, phantom fields require that the equation of state parameter $w^{1}$ should satisfy $w<-1$. Putting a - in front of the scalar field kinetic energy term can give $w<-1$, as in the original work on phantom fields by Caldwell [23], but this is not necessarily true. We will find that for most of our systems that our scalar fields are phantom (i.e., $w<-1$ ) for some range of coordinates, but will be nonphantom (i.e., $w>-1)$ for other ranges of coordinates. The corresponding energy-momentum tensor will then be

$$
\begin{aligned}
T_{\mu}^{\nu}= & -\left\{\partial_{\mu} \phi \partial^{\nu} \phi+\partial_{\mu} \chi \partial^{\nu} \chi\right. \\
& \left.-\delta_{\mu}^{\nu}\left[\frac{1}{2} \partial_{\mu} \phi \partial^{\mu} \phi+\frac{1}{2} \partial_{\mu} \chi \partial^{\mu} \chi-V(\phi, \chi)\right]\right\} .
\end{aligned}
$$

Since $T_{0}^{0}=\rho$ and $T_{i}^{i}=-p$, we can use (2) to calculate the equation of state parameter $w=\frac{p}{\rho}=-\frac{T_{i}^{i}}{T_{0}^{0}}$. As mentioned above, we will find (for three of the four systems studied) that the $w<-1$ for some range of spatial coordinates while being $w>-1$ for other ranges. Thus, the scalar fields, $\phi, \chi$ are partially phantom fields in that they satisfy $w<-1$ for some range of coordinates, but do not give $w<-1$ everywhere, and variation of the Lagrangian (1) gives the Einstein and scalar field equations in the form

$$
\begin{gathered}
G_{\mu}^{\nu}=\kappa T_{\mu}^{\nu}, \\
\frac{1}{\sqrt{-g}} \frac{\partial}{\partial x^{\mu}}\left[\sqrt{-g} g^{\mu \nu} \frac{\partial(\phi, \chi)}{\partial x^{\nu}}\right]=-\frac{\partial V}{\partial(\phi, \chi)},
\end{gathered}
$$

where $\kappa=8 \pi G$. Below we will consider only static problems, for which Eqs. (3)-(4) give the system of

\footnotetext{
${ }^{1}$ The equation of state parameter is the ratio of the pressure to the energy density of the field or fluid, namely, $w \equiv \frac{p}{\rho}$.
} 
ordinary nonlinear differential equations with the potential energy

$V(\phi, \chi)=\frac{\lambda_{1}}{4}\left(\phi^{2}-m_{1}^{2}\right)^{2}+\frac{\lambda_{2}}{4}\left(\chi^{2}-m_{2}^{2}\right)^{2}+\phi^{2} \chi^{2}-V_{0}$.

Here $m_{1}$ and $m_{2}$ are the masses of the scalar fields, $\lambda_{1}, \lambda_{2}$ are the coupling constants, and $V_{0}$ is some constant whose value will be given for each of the four types of solutions considered below. Our previous investigations of such kind of systems [17-22] indicate that regular solutions of the system (3)-(4) with the potential (5) do exist only for certain eigenvalues of the parameters $m_{1}, m_{2}$. We will find the same result here-that only certain values of $m_{1}, m_{2}$ lead to regular solutions.

In the following four sections, we will present the details of the four different kind of phantom field solutionsplanar "phantom" domain wall, spherically symmetric phantom ball, phantom wormhole and cylindrical phantom cosmic string. Many of the comments concerning the physical viability of these various solutions (or not) we will save for the concluding section.

\section{III. “PHANTOM” DOMAIN WALLS}

We begin by studying the simplest extended solution supported by two phantom scalar fields-the domain wall. The reason for the scare quotes around "phantom" in the section heading is due to the fact that, although our two scalar fields have the wrong sign in front of their kinetic energy terms, the equation of state parameter for these domain walls will never be less then -1 as we show at the end of this section. Since domain walls have planar symmetry, we choose the metric in the following form:

$$
d s^{2}=a^{2}(x)\left(d t^{2}-d y^{2}-d z^{2}\right)-d x^{2} .
$$

The metric function $a(x)$ depends on the coordinate $x$ only. This metric describes a two-dimensional domain wall embedded in a $(3+1)$-dimensional spacetime. Using Eqs. (2), (3), and (4), one can derive the following Einstein and scalar field equations:

$$
\begin{gathered}
3\left(\frac{a^{\prime}}{a}\right)^{2}=-\frac{1}{2}\left(\phi^{\prime 2}+\chi^{\prime 2}\right)+V, \\
\frac{a^{\prime \prime}}{a}-\left(\frac{a^{\prime}}{a}\right)^{2}=\frac{1}{2}\left(\phi^{\prime 2}+\chi^{\prime 2}\right),
\end{gathered}
$$

$$
\begin{aligned}
\phi^{\prime \prime}+3 \frac{a^{\prime}}{a} \phi^{\prime} & =\phi\left[2 \chi^{2}+\lambda_{1}\left(\phi^{2}-m_{1}^{2}\right)\right], \\
\chi^{\prime \prime}+3 \frac{a^{\prime}}{a} \chi^{\prime} & =\chi\left[2 \phi^{2}+\lambda_{2}\left(\chi^{2}-m_{2}^{2}\right)\right] .
\end{aligned}
$$

Here we have absorbed $\kappa$ via the following rescaling: $x / \sqrt{\kappa} \rightarrow x, \phi \sqrt{\kappa} \rightarrow \phi, \chi \sqrt{\kappa} \rightarrow \chi$, and $m_{1,2} \sqrt{\kappa} \rightarrow m_{1,2}$. The primes denote differentiation with respect to $x$. Looking at (7) and (8), one can see that these are essentially the Friedman equations but with the role of space and time coordinates exchanged (i.e., $t \leftrightarrow x$ ). The constant from the potential (5) is chosen to be

$V_{0}=\frac{\lambda_{1}}{4}\left[\phi^{2}(0)-m_{1}^{2}\right]^{2}+\frac{\lambda_{2}}{4}\left[\chi^{2}(0)-m_{2}^{2}\right]^{2}+\phi^{2}(0) \chi^{2}(0)$,

where the initial values, $\phi(0)$ and $\chi(0)$, are given below. From (7) one can see that this choice for $V_{0}$ ensures that $a^{\prime}(0)=0$. From (2) and for the metric in (6), the energy density is

$$
T_{0}^{0}=-\frac{\phi^{\prime 2}+\chi^{\prime 2}}{2}-V(\phi, \chi)
$$

We choose the boundary conditions at $x=0$ in the following form:

$$
\begin{aligned}
& \phi(0)=\sqrt{3}, \quad \phi^{\prime}(0)=0, \\
& \chi(0)=0.4,0.6,0.8,1.1,1.3 \quad \chi^{\prime}(0)=0, \\
& a(0)=1.0 .
\end{aligned}
$$

Following the procedure for finding solutions of Refs. $[18,20]$, we found the masses $m_{1,2}$ presented in Table I.

The results of numerical calculations for the scalar fields are given in Figs. 1 and 2; for the metric functions $a(x)$, see Fig. 3, and for the energy density $T_{0}^{0}$, see Fig. 4.

Let us estimate an asymptotic behavior of the solutions. One can see from Eq. (8) that the right-hand side goes asymptotically to zero, and the solution of this equation is

$$
a(x) \approx a_{0} e^{\alpha x}
$$

where $a_{0}$ and $\alpha$ are integration constants. This solution corresponds to an anti-de Sitter-like solution for the spatial

TABLE I. The eigenvalues $m_{1,2}$ versus $\chi(0)$ with $\phi(0)=\sqrt{3}$ for domain walls. The coupling constants are $\lambda_{1}=0.1$ and $\lambda_{2}=1$.

\begin{tabular}{lccccc}
\hline \hline$\chi(0)$ & 0.4 & 0.6 & 0.8 & 1.1 & 1.3 \\
\hline$m_{1}$ & 2.143597496 & 2.406090154 & 2.58469019 & 2.660693093 & 2.614816239 \\
$m_{2}$ & 2.64382729 & 2.796605957 & 2.98694478 & 3.348871789 & 3.655616217 \\
\hline \hline
\end{tabular}


VLADIMIR DZHUNUSHALIEV et al.

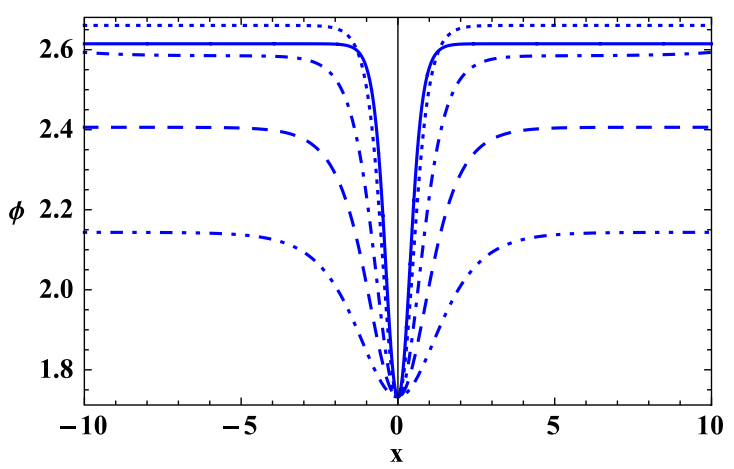

FIG. 1. The scalar fields $\phi(x)$ for the domain walls. In Figs. 1-4, the solid curve corresponds to $\chi(0)=1.3$, the dotted curve corresponds to $\chi(0)=1.1$, the dashed-dotted corresponds to $\chi(0)=0.8$, the dashed curve corresponds to $\chi(0)=0.6$, and the dash-double dotted curve corresponds to $\chi(0)=0.4$.

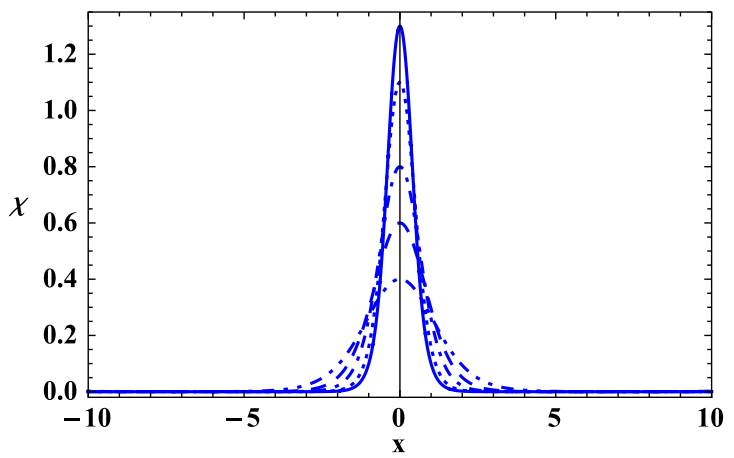

FIG. 2. The scalar fields $\chi(x)$ for the domain walls.

variable $x$. Then, using (14) we look for the asymptotic form of the scalar fields from Eqs. (9)-(10). Asymptotically we find that $\phi$ approaches $m_{1}$ and $\chi$ approaches zero so we write the field $\phi$ as $\phi \approx m_{1}-\delta \phi$ where $\delta \phi \ll 1$ as $x \rightarrow \pm \infty$. In this way, we obtain from Eqs. (9)-(10) the asymptotic equations for $\delta \varphi$ and $\chi$ :

$$
\begin{gathered}
\delta \phi^{\prime \prime}+3 \alpha \delta \phi^{\prime} \approx 2 \lambda_{1} m_{1}^{2} \delta \phi, \\
\chi^{\prime \prime}+3 \alpha \chi^{\prime} \approx\left(2 m_{1}^{2}-\lambda_{2} m_{2}^{2}\right) \chi .
\end{gathered}
$$

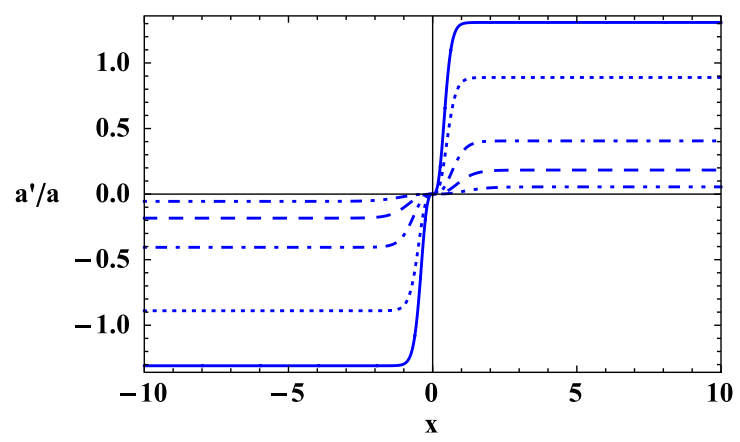

FIG. 3. The functions $a^{\prime}(x) / a(x)$ for the domain walls.
PHYSICAL REVIEW D 94, 024004 (2016)

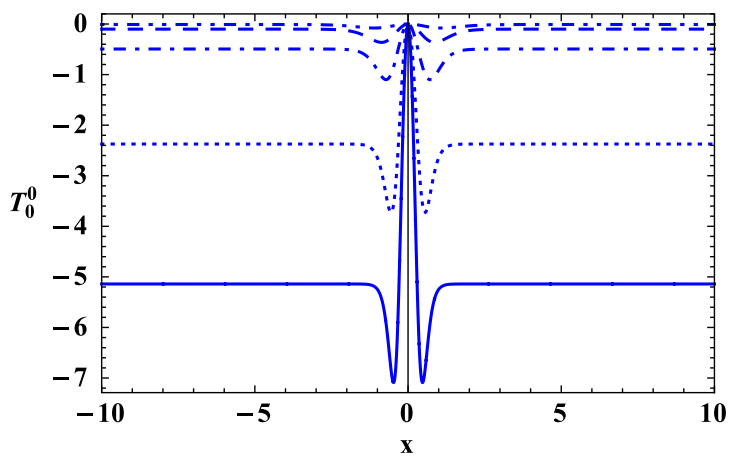

FIG. 4. The energy densities $T_{0}^{0}(x)$ for the domain walls.

The damping solutions to these equations are

$$
\begin{array}{r}
\delta \phi \approx C_{\phi} \exp \left[-\frac{x}{2}\left(3 \alpha+\sqrt{9 \alpha^{2}+8 \lambda_{1} m_{1}^{2}}\right)\right], \\
\chi \approx C_{\chi} \exp \left[-\frac{x}{2}\left(3 \alpha+\sqrt{9 \alpha^{2}+4\left(2 m_{1}^{2}-\lambda_{2} m_{2}^{2}\right)}\right)\right],
\end{array}
$$

where $C_{\phi}, C_{\chi}$ are integration constants. So we have solutions that tend asymptotically to the local minimum of the potential (5) at $\varphi=m_{1}$ and $\chi=0$. Note that with this asymptotic form of the scalar fields and for the choice of potential constant $V_{0}$ from (11) asymptotically $T_{0}^{0}<0$ as seen in Fig. 4. Actually $T_{0}^{0}<0$ for the entire range of $x$. While potentially unphysical, this negative energy density is not unexpected since in the case of some other types of planar solution [24], one also finds $T_{0}^{0}<0$.

Finally, in Fig. 5, we have given the equation of state parameter which is the ratio of the pressure to energy density of the field, namely, $w=\frac{p_{x}}{\rho}=-\frac{T_{1}^{1}}{T_{0}^{0}}$ where $p_{x}=$ $-T_{1}^{1}=-\left(\frac{\phi^{2}+\chi^{\prime 2}}{2}-V\right)$ and $\rho=T_{0}^{0}=-\frac{\phi^{12}+\chi^{\prime 2}}{2}-V$. The subscript $x$ indicates this is the pressure in the $x$ direction. The different components of the scalar field energy-momentum tensor are calculated via (2). There are also equation of state parameters for the two directions perpendicular to $x$ which are determined via the pressures, $-T_{2}^{2}$ and $-T_{3}^{3}$. Since we only assumed $x$ dependence, these two perpendicular

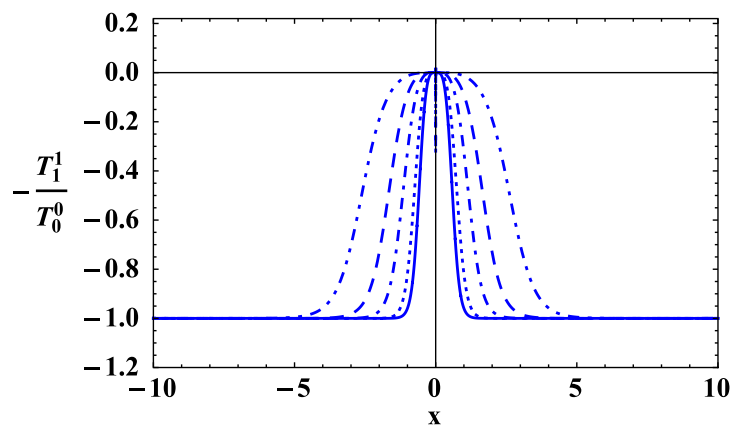

FIG. 5. The $x$-direction equation of state for the domain wall, $w=p_{x}(x) / \epsilon(x)$. 
equation of state parameters are identically equal to -1 , i.e., $-\frac{T_{2}^{2}}{T_{0}^{0}}=-\frac{T_{3}^{3}}{T_{0}^{0}}=-1$. In contrast, the radial equation of state parameter, $w=-\frac{T_{1}^{1}}{T_{0}^{0}}$, has more interesting behavior as shown in Fig. 5-it starts at $w=0$ at $x=0$ and asymptotically approaches -1 . Thus, for this case, the scalar fields are not phantom fields since $w \geq-1$ for all $x$. Thus, one cannot call these phantom domain walls. This is the reason for the scare quotes in the section heading.

\section{PHANTOM BALL}

In looking for spherically symmetric solutions of the above system of gravity plus two phantom scalar fields, with the requirement of trivial topology, we find that unlike our previous study [5] there are no boson star solutions. We do find spherically symmetric solution but the "mass" is of the order of the Planck mass rather than of astrophysical scale. Furthermore the mass of these solutions (obtained by integrating their energy density) leads to negative masses, making the physical use of these solutions extremely questionable. In our previous study, we assumed spherically symmetric solutions supported by one (complex) scalar field. Here we consider such spherically symmetric configurations created by two real phantom scalar fields.

To do so, let us choose the metric in Schwarzschild coordinates:

$$
d s^{2}=B(r) d t^{2}-A(r) d r^{2}-r^{2}\left(d \theta^{2}+\sin ^{2} \theta d \varphi^{2}\right) .
$$

Then the Einstein equations (3) and the scalar field equations (4) will be

$$
\begin{gathered}
\frac{1}{r} \frac{A^{\prime}}{A^{2}}+\frac{1}{r^{2}}\left(1-\frac{1}{A}\right)=-\frac{1}{2 A}\left(\phi^{\prime 2}+\chi^{\prime 2}\right)-V(\phi, \chi), \\
\frac{1}{r} \frac{B^{\prime}}{A B}-\frac{1}{r^{2}}\left(1-\frac{1}{A}\right)=-\frac{1}{2 A}\left(\phi^{\prime 2}+\chi^{\prime 2}\right)+V(\phi, \chi), \\
\frac{B^{\prime \prime}}{B}-\frac{1}{2}\left(\frac{B^{\prime}}{B}\right)^{2}-\frac{1}{2} \frac{A^{\prime}}{A} \frac{B^{\prime}}{B}-\frac{1}{r}\left(\frac{A^{\prime}}{A}-\frac{B^{\prime}}{B}\right) \\
=2 A\left[\frac{1}{2 A}\left(\phi^{\prime 2}+\chi^{\prime 2}\right)+V(\phi, \chi)\right], \\
\phi^{\prime \prime}+\left(\frac{2}{r}+\frac{B^{\prime}}{2 B}-\frac{A^{\prime}}{2 A}\right) \phi^{\prime}=A \phi\left[2 \chi^{2}+\lambda_{1}\left(\phi^{2}-m_{1}^{2}\right)\right], \\
\chi^{\prime \prime}+\left(\frac{2}{r}+\frac{B^{\prime}}{2 B}-\frac{A^{\prime}}{2 A}\right) \chi^{\prime}=A \chi\left[2 \phi^{2}+\lambda_{2}\left(\chi^{2}-m_{2}^{2}\right)\right] .
\end{gathered}
$$

In order to numerically solve the above system of coupled equations, we have introduced dimensionless variables via the following definitions: $r / \sqrt{\kappa} \rightarrow r, \phi \sqrt{\kappa} \rightarrow \phi, \chi \sqrt{\kappa} \rightarrow \chi$, and $m_{1,2} \sqrt{\kappa} \rightarrow m_{1,2}$. The primes denote differentiation with respect to the rescaled $r$. Since from the solutions below we find that the asymptotic values of the scalar fields are $\phi=$ $m_{1}$ and $\chi=0$ we pick the constant in the potential (5) as $V_{0}=(1 / 4) \lambda_{2} m_{2}^{4}$. This has results in the energy density being equal zero as $r \rightarrow \infty$. Equation (22) is a consequence of Eqs. (20) and (21), so we only have to solve the latter two equations for the metric functions $A(r), B(r)$.

Using Eqs. (20)-(24), one can find the following asymptotic behavior of the metric functions $A(r), B(r)$ and phantom scalar fields $\phi, \chi$ :

$$
\begin{gathered}
A \approx 1-\frac{r_{0}}{r}, \\
B \approx B_{\infty}\left(1+\frac{r_{0}}{r}\right), \\
\phi \approx m_{1}-C_{\phi} \frac{\exp \left(-\sqrt{2 \lambda_{1} m_{1}^{2}} r\right)}{r}, \\
\chi \approx C_{\chi} \frac{\exp \left(-\sqrt{\left(2 m_{1}^{2}-\lambda_{2} m_{2}^{2}\right)} r\right)}{r},
\end{gathered}
$$

where $r_{0}$ and $B_{\infty}$ are constants; $C_{\phi}, C_{\chi}$ are integration constants. In effect, $r_{0}$ defines the total mass of the system and $B_{\infty}$ - the rate of flow of time at infinity. By rescaling the time variable $t$, we can provide $B_{\infty}=1$ as $r \rightarrow \infty$, i.e., asymptotically we have flat Minkowski spacetime.

Here we expand on the studies of the spherically symmetric solutions performed in Ref. [18]. For this purpose, we will consider the properties of solutions depending on the central value of the scalar field $\chi_{0}$. The boundary conditions at $r=0$, for starting our numerical integration, are taken in the form:

$$
\begin{aligned}
& \phi(0)=0.5, \quad \phi^{\prime}(0)=0, \\
& \chi(0)=0.2,0.5,0.8,1.0,1.2, \quad \chi^{\prime}(0)=0, \\
& A(0)=1.0, \quad B(0)=1.0 .
\end{aligned}
$$

Since (20) and (21) are first order in the derivative we only need $A(0), B(0)$. Recall that (22) is redundant so in numerically solving the coupled system we only use (20), (21), (23), and (24). For a given value of $\chi(0)$ there are only certain values of $m_{1}, m_{2}$ which lead to regular solutions. These values of $m_{1}, m_{2}$ are found via the procedure described in $[18,20]$. The masses $m_{1,2}$ which yield regular solutions are shown in Table II as a function of $\chi(0)$. One may think of $m_{1}, m_{2}$ as eigenvalues.

TABLE II. The eigenvalues $m_{1,2}$ versus $\chi(0)$ with $\phi(0)=0.5$ for phantom ball solutions. The coupling constants are taken as $\lambda_{1}=0.1, \lambda_{2}=1$.

\begin{tabular}{cccccc}
\hline \hline$\chi(0)$ & 0.2 & 0.5 & 0.8 & 1. & 1.2 \\
\hline$m_{1}$ & 0.64929 & 0.941025 & 1.26 & 1.4882 & 1.73298 \\
$m_{2}$ & 0.86731 & 1.14404 & 1.42583 & 1.61421 & 1.80206 \\
\hline \hline
\end{tabular}




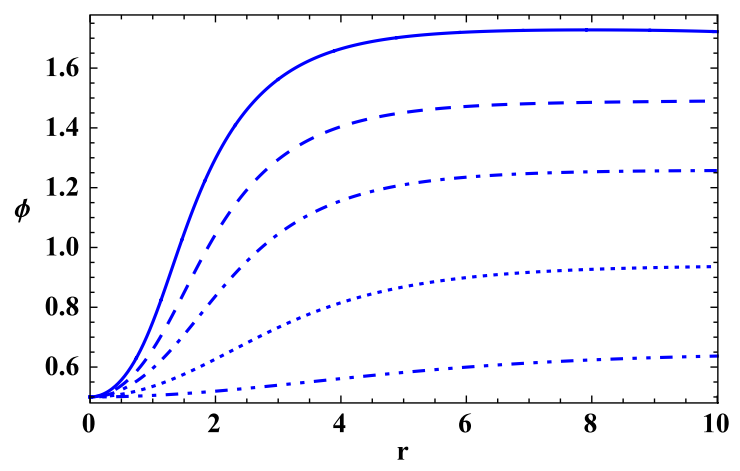

FIG. 6. The scalar fields $\phi(r)$ for the phantom ball. In Figs. 6-12, the solid curve corresponds to $\chi(0)=1.2$, the dashed curve corresponds to $\chi(0)=1.0$, the dashed-dotted corresponds to $\chi(0)=0.8$, the dotted curve corresponds to $\chi(0)=0.5$, and the dash-double dotted curve corresponds to $\chi(0)=0.2$.

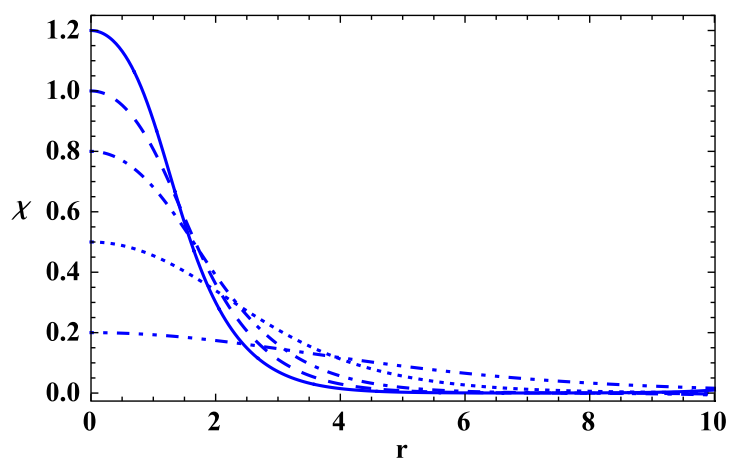

FIG. 7. The scalar fields $\chi(r)$ for the phantom ball.

The results of the numerical calculations for the scalar fields are presented in Figs. 6 and 7; for the metric functions $A(r), B(r)$, see Figs. 8 and 9, and for the energy density $T_{0}^{0}$, see Fig. 10.

In Fig. 11, we have plotted the radial equation of state parameter, $w=\frac{p_{r}}{\rho}=-\frac{T_{1}^{1}}{T_{0}^{0}}$, for the phantom ball. As was the case with the domain wall, the equation of state parameters for the two perpendicular directions (i.e., $w=\frac{p_{\theta}}{\rho}=-\frac{T_{2}^{2}}{T_{0}^{0}}$

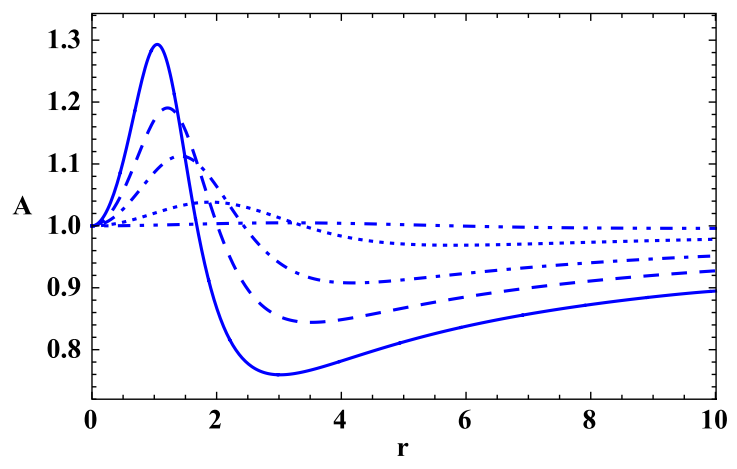

FIG. 8. The metric functions $A(r)$ for the phantom ball.

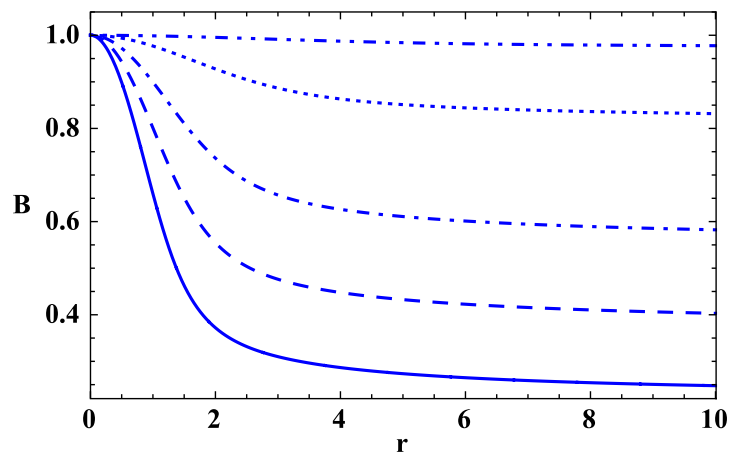

FIG. 9. The metric functions $B(r)$ for the phantom ball.

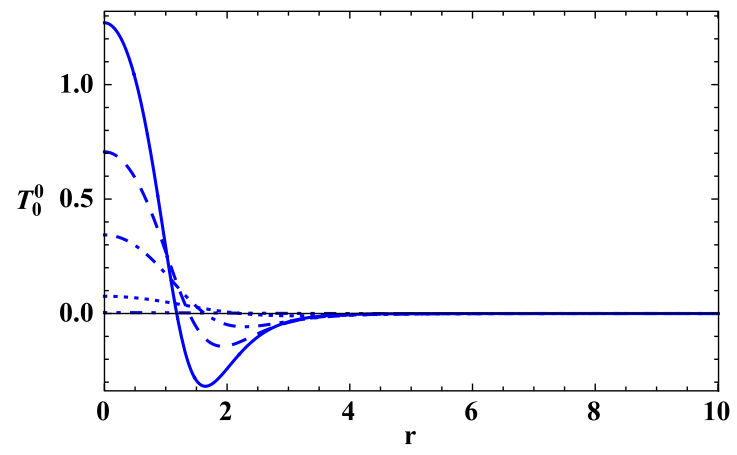

FIG. 10. The energy density profiles for the phantom ball.

and $\left.w=\frac{p_{\varphi}}{\rho}=-\frac{T_{3}^{3}}{T_{0}^{0}}\right)$ are identically equal to -1 . The divergence of $w$ seen in Fig. 11 comes from the places where $T_{0}^{0} \rightarrow 0$ as can be seen in Fig. 10 .

From the equation of state parameter in Fig. 11, we find that for some range of $r$ near the origin the two scalar fields are phantom fields with $w<-1$, but for $r \rightarrow \infty$ the scalar fields have less exotic equations of state and for some parameters go to the dust equation of state $w=0$. It is because of the phantom behavior near $r=0$ that we call these solutions "phantom ball" solutions. To further examine the physical character of these solutions, we turn to their total mass. We begin by defining the dimensionless mass of a phantom ball as

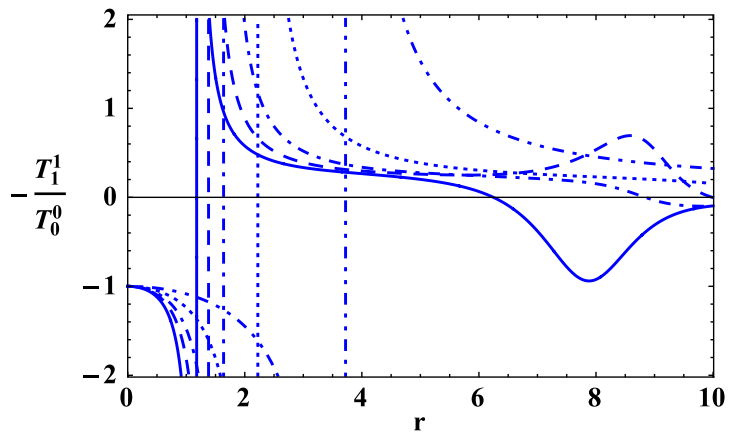

FIG. 11. The radial equation of state for the phantom ball $w=p_{r}(r) / \epsilon(r)$. 


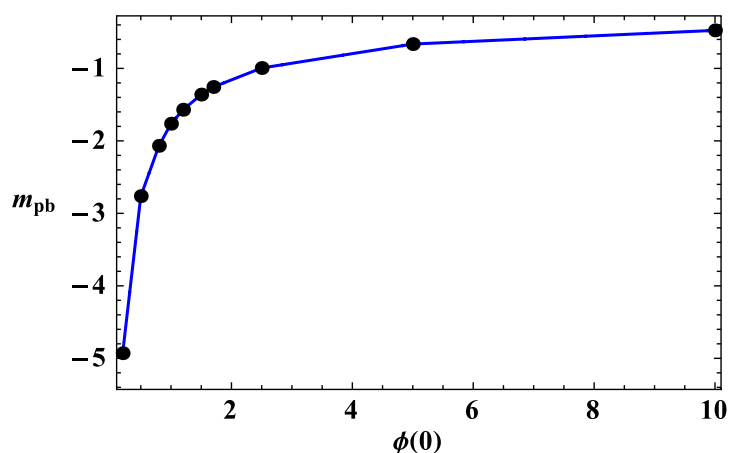

FIG. 12. The mass of the phantom ball for $\chi(0)=0.5$ and as a function of $\phi(0)=0.2,0.5,0.8,1 ., 1.2,1.5,1.7,2.5,5,10$.

$$
\begin{gathered}
m_{p b}=4 \pi \int_{0}^{\infty} r^{2} \sqrt{A(r)} \epsilon_{p b} d r \\
\epsilon_{p b} \equiv T_{0}^{0}=-\frac{\phi^{\prime 2}+\chi^{\prime 2}}{2 A}-V(\phi, \chi),
\end{gathered}
$$

where $\epsilon_{p b}$ is the total energy density. This corresponds to the proper mass of the system [25] which is the Newtonian mass of the system (i.e., $4 \pi \int_{0}^{\infty} r^{2} \epsilon_{p b} d r$ ) plus the binding energy. The profile of $m_{p b}$ as a function of $\phi(0)$ for $\chi(0)=$ 0.5 is presented in Fig. 12. The values of $\phi(0)$ are $\phi(0)=0.2,0.5,0.8,1 ., 1.2,1.5,1.7,2.5,5,10$. For each different $\phi(0)$ we needed to calculate the associated masses $m_{1}, m_{2}$. The main thing to note is that $m_{p b}<0$ which makes the physical use of these solutions questionable. Also, taking into account the rescaling that was performed on the coordinates, fields and parameter, the magnitude of $m_{p b}$ is of the order of the Planck mass. Thus, even if $m_{p b}$ were positive, these would not be astrophysical solutions but would be a Planck mass particlelike solution. One could compare the phantom ball solutions to GUT magnetic monopole solutions [26]. Unlike GUT monopoles the present solutions do not involve gauge fields. In the next section, by changing our metric form from Schwarzschildlike to wormholelike, we do find positive mass solutions for some parameters.

\section{PHANTOM TRAVERSABLE WORMHOLES}

We seek static solutions of Eqs. (3)-(4) for the wormhole metric in polar Gaussian coordinates [27],

$$
d s^{2}=B(r) d t^{2}-d r^{2}-A(r)\left(d \theta^{2}+\sin ^{2} \theta d \varphi^{2}\right),
$$

where $A(r), B(r)$ are even functions depending only on the coordinate $r$ which covers the entire range $-\infty<r<+\infty$. Using this metric, one can obtain the following complete system of the Einstein and scalar field equations:

$$
\begin{gathered}
\frac{A^{\prime \prime}}{A}-\frac{1}{2}\left(\frac{A^{\prime}}{A}\right)^{2}-\frac{1}{2} \frac{A^{\prime}}{A} \frac{B^{\prime}}{B}=\phi^{2}+\chi^{\prime 2}, \\
\frac{A^{\prime \prime}}{A}+\frac{1}{2} \frac{A^{\prime}}{A} \frac{B^{\prime}}{B}-\frac{1}{2}\left(\frac{A^{\prime}}{A}\right)^{2}-\frac{1}{2}\left(\frac{B^{\prime}}{B}\right)^{2}+\frac{B^{\prime \prime}}{B} \\
=2\left[\frac{1}{2}\left(\phi^{\prime 2}+\chi^{\prime 2}\right)+V\right], \\
\frac{1}{4}\left(\frac{A^{\prime}}{A}\right)^{2}-\frac{1}{A}+\frac{1}{2} \frac{A^{\prime}}{A} \frac{B^{\prime}}{B}=-\frac{1}{2}\left(\phi^{\prime 2}+\chi^{\prime 2}\right)+V, \\
\phi^{\prime \prime}+\left(\frac{A^{\prime}}{A}+\frac{1}{2} \frac{B^{\prime}}{B}\right) \phi^{\prime}=\phi\left[2 \chi^{2}+\lambda_{1}\left(\phi^{2}-m_{1}^{2}\right)\right], \\
\chi^{\prime \prime}+\left(\frac{A^{\prime}}{A}+\frac{1}{2} \frac{B^{\prime}}{B}\right) \chi^{\prime}=\chi\left[2 \phi^{2}+\lambda_{2}\left(\chi^{2}-m_{2}^{2}\right)\right],
\end{gathered}
$$

where the prime denotes differentiation with respect to $r$. Eq. (33) is obtained by subtracting the $\left(\begin{array}{l}r \\ r\end{array}\right)$ component of Eqs. (3) from the $\left(\begin{array}{l}t \\ t\end{array}\right)$ component, and Eqs. (34) and (35) are the $\left(\begin{array}{l}\theta \\ \theta\end{array}\right)$ and $\left(\begin{array}{l}r \\ r\end{array}\right)$ components of Eqs. (3). In Eqs. (33)-(37), the following rescaling has been introduced: $r \rightarrow \sqrt{\kappa} r$, $\varphi \rightarrow \varphi / \sqrt{\kappa}, \chi \rightarrow \chi / \sqrt{\kappa}, m_{1,2} \rightarrow m_{1,2} / \sqrt{\kappa}, A(r) \rightarrow 8 \pi G A(r)$. The arbitrary potential constant was chosen as $V_{0}=$ $\left(\lambda_{2} / 4\right) m_{2}^{4}$ so that the value of the potential in the local minimum (i.e., $\phi=m_{1}$ and $\chi=0$ ) was equal to zero- $V\left(\phi=m_{1}, \chi=0\right)=0$. This choice of $V_{0}$ also ensures zero value of the energy density as $r \rightarrow \pm \infty$ [see Fig. 17].

Taking into account the $\mathbb{Z}_{2}$ symmetry of the problem, the boundary conditions are chosen in the following form:

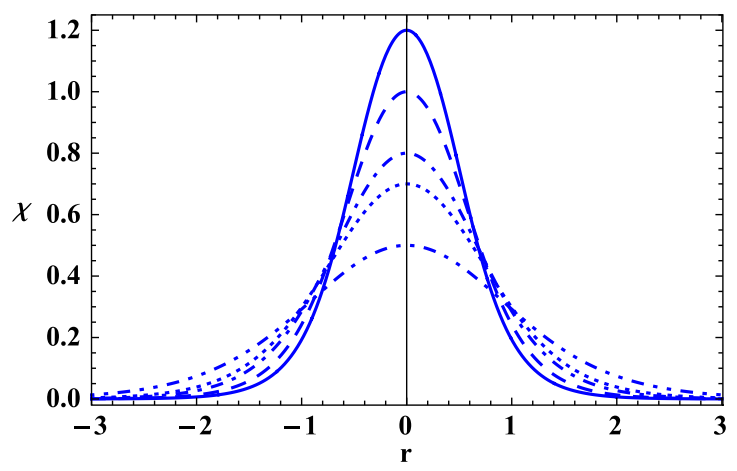

FIG. 13. The behavior of the scalar field $\chi(r)$ for the traversable wormhole. In Figs. 13-17, the solid curve corresponds to $\chi(0)=1.2$, the dashed curve corresponds to $\chi(0)=1.0$, the dashed-dotted corresponds to $\chi(0)=0.8$, the dotted curve corresponds to $\chi(0)=0.7$, and the dash-double dotted curve corresponds to $\chi(0)=0.5$. 


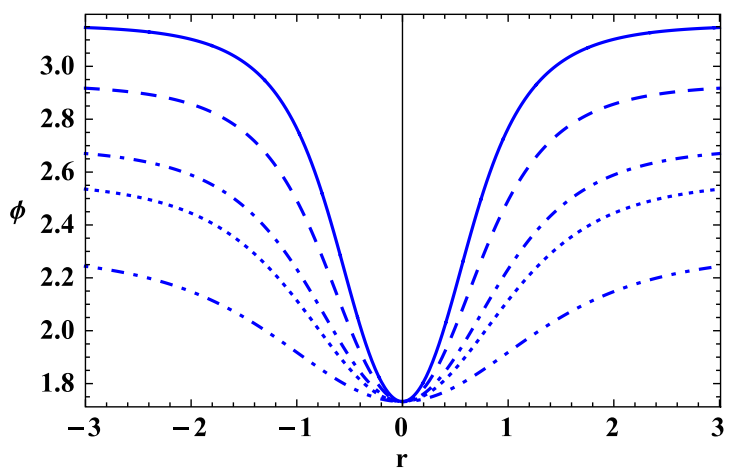

FIG. 14. The behavior of the scalar field $\phi(r)$ for the traversable wormhole.

$$
\begin{aligned}
& \phi(0)=\sqrt{3}, \quad \phi^{\prime}(0)=0, \\
& \chi(0)=0.5,0.7,0.8,1.0,1.2, \quad \chi^{\prime}(0)=0, \\
& A(0)=-\frac{1}{V(\phi(0), \chi(0))}, \quad A^{\prime}(0)=0, \\
& B(0)=1.0, \quad B^{\prime}(0)=0,
\end{aligned}
$$

where the condition for $A(0)$ is chosen so as to satisfy the constraint (35) at $r=0, V(\phi(0), \chi(0))$ is the value of the potential at $r=0$, and the coupling constants are $\lambda_{1}=0.1$ and $\lambda_{2}=1$. Then, using the procedure for finding solutions of Refs. [18,20], we obtained the results presented in Figs. 13-19. The values of the masses $m_{1,2}$ for the five values of $\chi(0)$ from (38) are given in Table III.

The asymptotic behavior of the solutions is

$$
\begin{gathered}
A \approx r^{2}+r_{0}^{2}, \\
B \approx B_{\infty}\left(1-\frac{r_{0}^{2}}{r^{2}}\right), \\
\phi \approx m_{1}-C_{\phi} \frac{\exp \left(-r \sqrt{2 \lambda_{1} m_{1}^{2}}\right)}{r},
\end{gathered}
$$

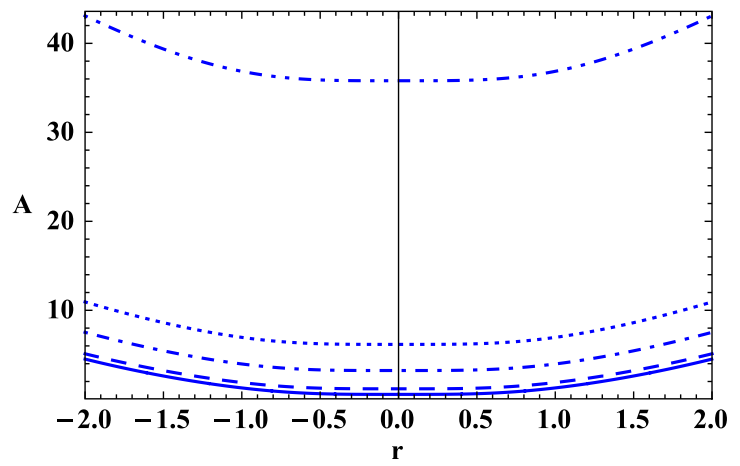

FIG. 15. The metric function $A(r)$ for the traversable wormhole.

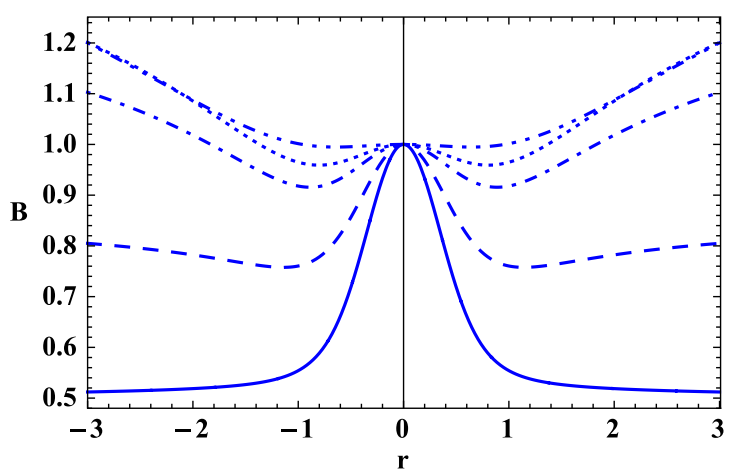

FIG. 16. The metric function $B(r)$ for the traversable wormhole.

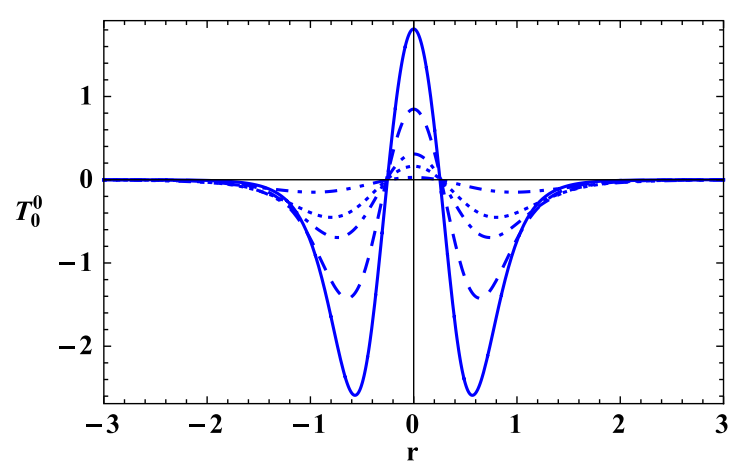

FIG. 17. The energy densities distributions for the traversable wormhole.

$$
\chi \approx C_{\chi} \frac{\exp \left(-r \sqrt{2 m_{1}^{2}-\lambda_{2} m_{2}^{2}}\right)}{r}
$$

where $r_{0}, B_{\infty}, C_{\phi}, C_{\chi}$ are integration constants.

In Fig. 18, we have plotted the equation of state parameter $w=\frac{p_{r}}{\rho}=-\frac{T_{1}^{1}}{T_{0}^{0}}$ for the $r$ direction (again the equation of state for the two perpendicular directions yields $w=\frac{p_{\theta}}{\rho}=-\frac{T_{2}^{2}}{T_{0}^{0}}=-1$ and $\left.w=\frac{p_{\varphi}}{\rho}=-\frac{T_{3}^{3}}{T_{0}^{0}}=-1\right)$. As with the phantom ball solutions there is a region near the origin

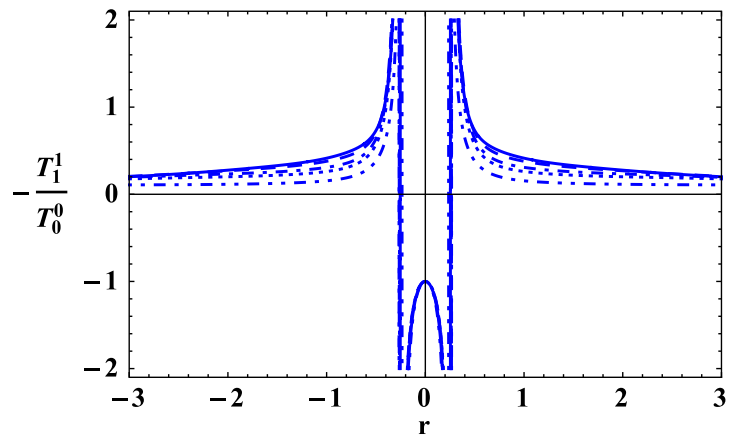

FIG. 18. The equation of state $w=p_{r}(r) / \epsilon(r)$ for the wormhole solutions. 


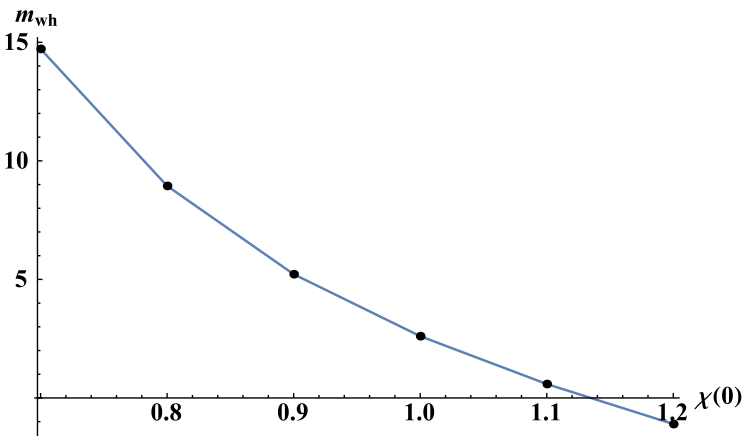

FIG. 19. Wormhole mass as a function of $\chi(0)=0.7,0.8,0.9$, $1.0,1.1,1.2$.

where the fields have a phantom equation of state with $w<-1$. The divergences in $w$ again come from the places where $T_{0}^{0}=0$ which can be seen in Fig. 17. Unlike the phantom ball solutions, we now show that the wormhole solutions do allow positive mass.

The mass of the wormhole is taken to be the effective mass defined in [27] as follows:

$$
m_{w h}(r)=\frac{R(0)}{2 G}+4 \pi \int_{R(0)}^{r} T_{0}^{0} R^{2} d R
$$

The mass in (43) uses a wormhole metric in the form

$$
d s^{2}=B(r) d t^{2}-d r^{2}-R^{2}(r)\left(d \theta^{2}+\sin ^{2} \theta d \varphi^{2}\right) .
$$

The ansatz function $R(r)$ in (44) is related to the metric ansatz function used in this work by $R(r)=\sqrt{A(r)}$. In the limit $r \rightarrow \infty$, the wormhole mass, for the form of the metric given in (32), becomes

$$
m_{w h}(\infty)=4 \pi \sqrt{A(0)}+2 \pi \int_{0}^{\infty} T_{0}^{0} \sqrt{A} \frac{d A}{d r} d r
$$

where the energy density is given as

$$
\begin{aligned}
T_{0}^{0}= & -\frac{\phi^{\prime 2}+\chi^{\prime 2}}{2}-\frac{\lambda_{1}}{4}\left(\phi^{2}-m_{1}^{2}\right)^{2} \\
& -\frac{\lambda_{2}}{4}\left(\chi^{4}-2 \chi^{2} m_{2}^{2}\right)-\phi^{2} \chi^{2},
\end{aligned}
$$

where we set $V_{0}=\frac{\lambda_{2}}{4} m_{2}^{4}$ to get the potential part energy density to have the form given in (46) The wormhole mass $m_{w h}(\infty)$ has been rescaled using the same rescaling of the masses, $m_{1,2}$, namely $m_{w h}(\infty) \rightarrow m_{w h}(\infty) / \sqrt{8 \pi G}$. The profile of $m_{w h}$ as a function of $\chi(0)$ is shown in Fig. 19.

\section{PHANTOM COSMIC STRINGS}

In this section, we consider the case of an extended one dimensional solution-the global cosmic string. Global cosmic strings are built from scalar fields only. In contrast, local cosmic string involve scalar fields plus gauge fields. This type of solution has cylindrical symmetry and we describe it via the following metric,

$$
\begin{aligned}
d s^{2}= & e^{2 \nu(\rho)} d t^{2}-e^{2(\gamma(\rho)-\psi(\rho))} d \rho^{2}-e^{2 \psi(\rho)} d z^{2} \\
& -\rho^{2} e^{-2 \psi(\rho)} d \varphi^{2}
\end{aligned}
$$

and the assumption that the scalar fields are only dependent on $\rho$. Substituting this into the Einstein and scalar field equations (3)-(4), one can obtain the following set of equations:

$$
\begin{gathered}
\frac{\gamma^{\prime}}{\rho}-\psi^{\prime 2}=-\kappa\left(\frac{1}{2} \phi^{\prime 2}+\frac{1}{2} \chi^{\prime 2}+e^{2(\gamma-\psi)} V(\phi, \chi)\right), \\
\frac{\nu^{\prime}+\psi^{\prime}}{\rho}-\psi^{\prime 2}=-\kappa\left(\frac{1}{2} \phi^{\prime 2}+\frac{1}{2} \chi^{\prime 2}-e^{2(\gamma-\psi)} V(\phi, \chi)\right),
\end{gathered}
$$

$$
\begin{gathered}
\psi^{\prime \prime}-\nu^{\prime \prime}-\psi^{\prime} \gamma^{\prime}+\nu^{\prime} \gamma^{\prime}-\nu^{\prime 2}+\frac{\psi^{\prime}+\gamma^{\prime}-\nu^{\prime}}{\rho} \\
=\kappa\left(-\frac{1}{2} \phi^{\prime 2}-\frac{1}{2} \chi^{\prime 2}-e^{2(\gamma-\psi)} V(\phi, \chi)\right), \\
-\psi^{\prime \prime}-\nu^{\prime \prime}+\psi^{\prime} \gamma^{\prime}+\nu^{\prime} \gamma^{\prime}-2 \psi^{\prime 2}-2 \psi^{\prime} \nu^{\prime}-\nu^{\prime 2} \\
=\kappa\left(-\frac{1}{2} \phi^{\prime 2}-\frac{1}{2} \chi^{\prime 2}-e^{2(\gamma-\psi)} V(\phi, \chi)\right), \\
\phi^{\prime \prime}+\phi^{\prime}\left(\frac{1}{\rho}-\gamma^{\prime}+\psi^{\prime}+\nu^{\prime}\right)=e^{2(\gamma-\psi)} \phi\left[2 \chi^{2}+\lambda_{1}\left(\phi^{2}-m_{1}^{2}\right)\right], \\
\chi^{\prime \prime}+\chi^{\prime}\left(\frac{1}{\rho}-\gamma^{\prime}+\psi^{\prime}+\nu^{\prime}\right)=e^{2(\gamma-\psi)} \chi\left[2 \phi^{2}+\lambda_{2}\left(\chi^{2}-m_{2}^{2}\right)\right] .
\end{gathered}
$$

As in the case of the phantom balls and the traversable wormhole, we have taken the potential constant as $V_{0}=$ $\left(\lambda_{2} / 4\right) m_{2}^{4}$ so that the potential will go to zero as $\rho \rightarrow \infty$.

TABLE III. The eigenvalues $m_{1,2}$ versus $\chi(0)$ with $\phi(0)=\sqrt{3}$ for phantom traversable wormholes.

\begin{tabular}{lllllr}
\hline \hline$\chi(0)$ & \multicolumn{1}{c}{0.5} & \multicolumn{1}{c}{0.7} & \multicolumn{1}{c}{0.8} & \multicolumn{1}{c}{1.0} & 1.2 \\
\hline$m_{1}$ & 2.2899327 & 2.565822825 & 2.693582264 & 2.931316954 & 3.155579948 \\
$m_{2}$ & 2.712082667 & 2.866518677 & 2.949800162 & 3.12411743 & 3.304034608 \\
\hline \hline
\end{tabular}


TABLE IV. The eigenvalues $m_{1,2}$ versus $\chi(0)$ with $\phi(0)=0.5$ for phantom cosmic strings. The coupling constants $\lambda_{1}=0.1$, $\lambda_{2}=1$.

\begin{tabular}{lcccc}
\hline \hline$\chi(0)$ & 0.1 & \multicolumn{1}{c}{0.4} & 0.6 & 0.8 \\
\hline$m_{1}$ & 1.7720039 & 2.044828 & 2.2706674 & 2.515715 \\
$m_{2}$ & 2.4896351 & 2.7060905 & 2.8649473 & 3.0277392 \\
\hline \hline
\end{tabular}

To simplify the above equations we also make the additional assumption that two of the metric functions are equal i.e., $\nu=\psi$. After some algebraic manipulations, and performing the rescaling $\rho / \sqrt{\kappa} \rightarrow \rho, \phi \sqrt{\kappa} \rightarrow \phi$, $\chi \sqrt{\kappa} \rightarrow \chi$, and $m_{1,2} \sqrt{\kappa} \rightarrow m_{1,2}$ we get the following equations for the metric functions $\gamma(\rho), \psi(\rho)$ and phantom scalar fields $\phi(\rho), \chi(\rho)$ :

$$
\begin{gathered}
\frac{\gamma^{\prime}}{\rho}-\psi^{\prime 2}=-\left(\frac{1}{2} \phi^{\prime 2}+\frac{1}{2} \chi^{\prime 2}+e^{2(\gamma-\psi)} V(\phi, \chi)\right), \\
2 \frac{\psi^{\prime}}{\rho}-\psi^{\prime 2}=-\left(\frac{1}{2} \phi^{\prime 2}+\frac{1}{2} \chi^{\prime 2}-e^{2(\gamma-\psi)} V(\phi, \chi)\right), \\
\psi^{\prime \prime}+\frac{\psi^{\prime}}{\rho}=e^{2(\gamma-\psi)}\left(1-2 \rho \psi^{\prime}\right) V(\phi, \chi),
\end{gathered}
$$

$\phi^{\prime \prime}+\phi^{\prime}\left(\frac{1}{\rho}-\gamma^{\prime}+2 \psi^{\prime}\right)=e^{2(\gamma-\psi)} \phi\left[2 \chi^{2}+\lambda_{1}\left(\phi^{2}-m_{1}^{2}\right)\right]$,

$\chi^{\prime \prime}+\chi^{\prime}\left(\frac{1}{\rho}-\gamma^{\prime}+2 \psi^{\prime}\right)=e^{2(\gamma-\psi)} \chi\left[2 \phi^{2}+\lambda_{2}\left(\chi^{2}-m_{2}^{2}\right)\right]$

where the prime denotes differentiation with respect to the rescaled radial coordinate $\rho$.

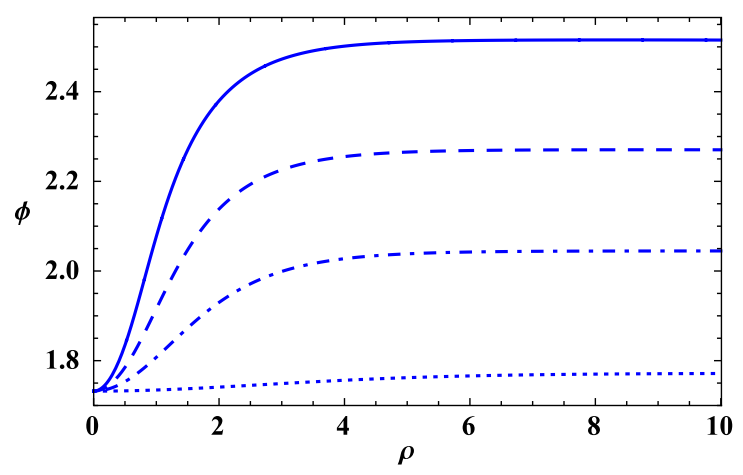

FIG. 20. The scalar fields $\phi(\rho)$ for the phantom cosmic strings. For Figs. 20-24, the solid curve corresponds to $\chi(0)=0.8$, the dashed curve corresponds to $\chi(0)=0.6$, the dashed-dotted corresponds to $\chi(0)=0.4$, and the dotted curve corresponds to $\chi(0)=0.1$.

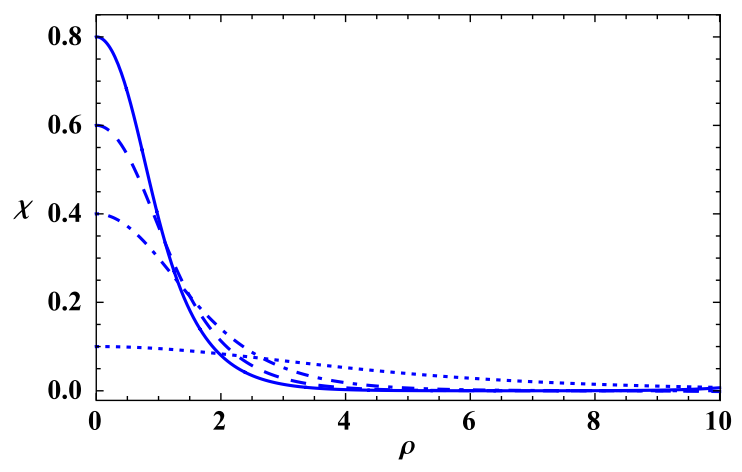

FIG. 21. The scalar fields $\chi(\rho)$ for the phantom cosmic strings.

To numerically integrate the above equations we will use (54), (56), (57), and (58), along with the definition of $V(\phi, \chi)$ in (5). Equation (55) is redundant. The boundary conditions are chosen at the center $(\rho=0)$ in the following form:

$$
\begin{aligned}
\gamma(0) & =0, \\
\psi(0) & =0, \quad \psi^{\prime}(0)=0, \\
\phi(0) & =\sqrt{3}, \quad \phi^{\prime}(0)=0, \\
\chi(0) & =0.1,0.4,0.6,0.8, \quad \chi^{\prime}(0)=0 .
\end{aligned}
$$

As before, for a given initial value of $\chi(0)$, there are special values (eigenvalues) for $m_{1}, m_{2}$ for which solutions are found with acceptable asymptotic behavior. The procedure for finding these special values of $m_{1}, m_{2}$ is that given in Refs. [18,20]. The value of these masses $m_{1}, m_{2}$ as a function of $\chi(0)$ is shown in Table IV.

The results of numerical calculations for the scalar fields, $\phi$ and $\chi$, are given in Figs. 20 and 21. The behavior of the scalar fields is similar to what was found for the phantom balls. The scalar field $\phi$ started at $\phi=0$ and as $\rho \rightarrow \infty$ approached some constant, nonzero value. The scalar field $\chi$ started at some nonzero value and approached $\chi=0$ as $\rho \rightarrow \infty$. The metric functions $\gamma(\rho), \psi(\rho)$ are given in Figs. 22 and 23. These metric functions both approach

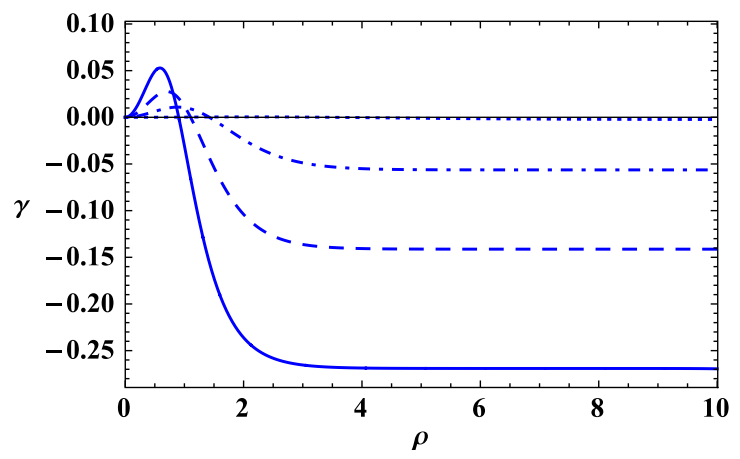

FIG. 22. The metric functions $\gamma(\rho)$ for the phantom cosmic strings. 


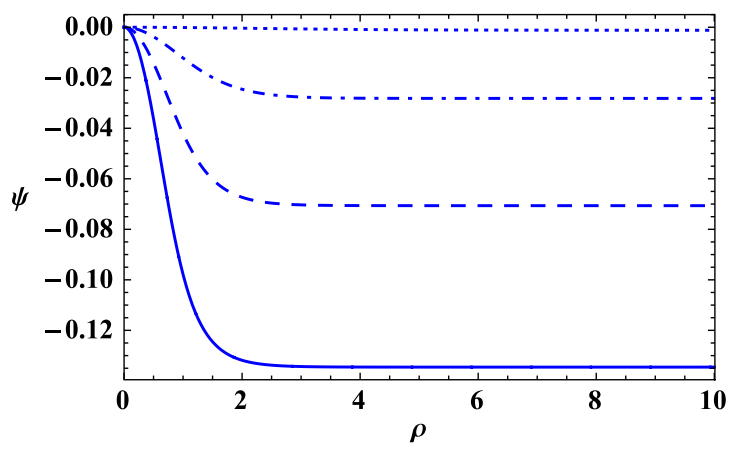

FIG. 23. The metric functions $\psi(\rho)$ for the phantom cosmic strings.

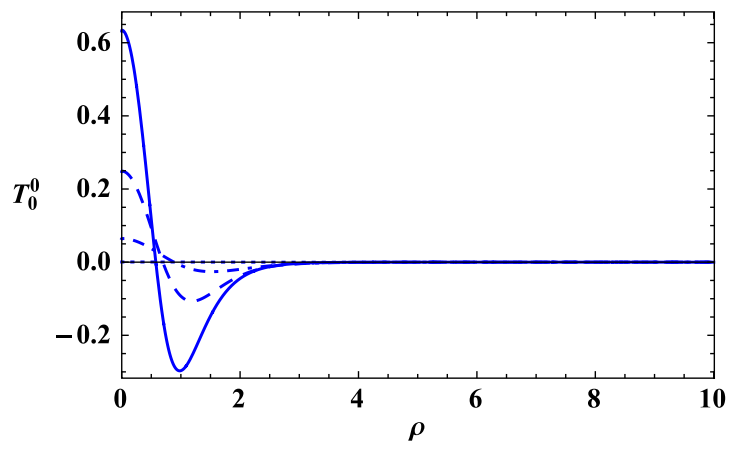

FIG. 24. The energy density profiles for the phantom cosmic strings.

some constant negative value as $\rho \rightarrow \infty$. The energy density, $T_{0}^{0}$, is given in Fig. 24. Near $\rho \approx 0.5$ the energy density, $T_{0}^{0}$, changes sign and then goes asymptotically to zero from below.

Since the cosmic string is of infinite length its total mass is infinite, but the relevant quantity is mass per unit length. To obtain this we integrate the energy density, $T_{0}^{0}$, over the full range of $\rho$ and $\varphi$ but along $z$ we integrate a finite length, $L$, and then divide by this to get mass per unit length. The energy density (2) is

$$
T_{0}^{0}=-\frac{1}{2}\left(\phi^{\prime 2}+\chi^{\prime 2}\right) e^{(2 \psi-2 \gamma)}-V(\phi, \chi) .
$$

Now the mass of a length $L$ of the cosmic string is

$$
m_{c s}=2 \pi L \int_{0}^{\infty} T_{0}^{0} \rho e^{(\gamma-\psi)} d \rho,
$$

where the $\varphi$ integration gives $2 \pi$ and the $z$ integration gives $L$. The factor $\rho e^{(\gamma-\psi)}$ comes from $\sqrt{-\operatorname{det} g^{3}}$, the volume factor for the spatial part of the metric in (47). From (61) and (60) the mass per unit length, $\mu_{c s} \equiv m_{c s} / L$, of the cosmic string solution is

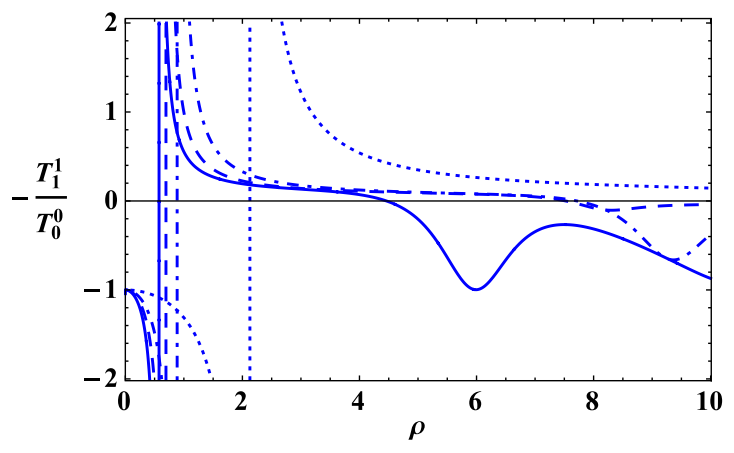

FIG. 25. The equation of state $w=p_{\rho}(\rho) / \epsilon(\rho)$ for the cosmic string solutions.

$$
\begin{aligned}
\mu_{c s} & =2 \pi \int_{0}^{\infty} T_{0}^{0} \rho e^{(\gamma-\psi)} d \rho \\
& =\int_{0}^{\infty}\left[-\frac{\rho}{2}\left(\phi^{\prime 2}+\chi^{\prime 2}\right) e^{(\psi-\gamma)}-\rho V(\phi, \chi) e^{(\gamma-\psi)}\right] d \rho .
\end{aligned}
$$

We calculated $\mu_{c s}$ for a range of different initial values of $\chi(0)$ and $\phi(0)$ and in general found that $\mu_{c s}<0$. In one case when $\phi(0)=0$ and $\chi(0)=0$, we did find by extrapolation that a very small positive value for $\mu_{c s}$. However, for this point we could not trust the extrapolation. Thus, in this case, the conclusion was that $\mu_{c s}<0$ in general.

The equation of state parameter, $w=-\frac{T_{1}^{1}}{T_{0}^{0}}$ for the cosmic string is shown in Fig. 25. As in the previous three cases, we have defined the equation of state parameter in terms of the radial pressure (for the cosmic string this means pressure in the $\rho$ direction). The equation of state parameter has some range of $\rho$ near $\rho=0$ for which $w<-1$ (i.e., the scalar fields have a phantom equation of state) and so we call these solutions phantom cosmic strings. As with the wormhole and phantom ball solutions the region where one has phantom behavior is near the origin. From Fig. 25, one can see that $w$ diverges. These divergences occur at the places where $T_{0}^{0} \rightarrow 0$ as shown in Fig. 24 .

\section{DISCUSSION AND CONCLUSIONS}

We have studied several hypothetical compact and extended astrophysical objects supported by two phantom scalar fields. When distributed homogeneously and isotropically over the Universe such phantom fields could be the source of the present accelerated expansion of the Universe. However, one can imagine situations when in the process of evolution of the Universe there could arise inhomogeneous distributions of such phantom fields that might lead to the formation of localized or partially localized configurations such as those considered here.

The "phantom" domain walls investigated in Sec. III are, in fact, not phantom since, despite having negative-sign kinetic energy terms for the scalar fields, the equation of state parameter associated with the $x$ direction is always 
$w \geq-1$ as shown in Fig. 5. Leaving aside the equation of state parameter, these domain wall solutions do have the "unphysical" characteristic that their energy density, $T_{0}^{0}$, is everywhere negative $T_{0}^{0}<0$. This leads one to strongly suspect that these solutions are unstable, i.e., that they would dissipate rapidly. This is actually a good, physical feature since normal domain walls tend to dominate the energy density of the Universe [28] and can be ruled out observationally. Thus, the domain walls studied here, exactly because of their hypothesized unstable nature, could be of more consequence than normal domain walls - these domain walls might form and quickly dissipate, perhaps leaving some small imprint on the CMB. In regards to observational constraints, regular domain walls are more problematic. However, one would still need to confirm that these domain walls are unstable by performing some stability analysis as in [29], and one would need to determine if they would leave some imprint on observables from the early Universe.

In Sec. IV, we found phantom ball solutions. In contrast to the domain wall solutions, the phantom ball solutions can properly be call "phantom solutions" since for some range of $r$ near the origin their equation of state parameter does satisfy $w<-1$ as seen in Fig. 11. These solutions might be viewed as phantom versions of magnetic monopole solutions [26] that occur in grand unified theories (GUT), except that the phantom ball solutions of Sec. IV involve scalar fields coupled to gravity, while the GUT monopoles are a combination of gauge and scalar fields. These GUT monopoles have been shown to be problematic to cosmological observations [30] (one can solve the "GUT monopole problem" via inflation). The phantom ball solutions had some range of $r$ where the energy density was positive, $T_{0}^{0}>0$ as seen in Fig. 10 . However, when integrating the energy density over all space we found that the mass of the phantom balls was negative as seen in Fig. 12. Thus, as with the domain walls, the phantom ball solutions are probably unstable (of course, for both solutions, one should check this in detail, but it would be odd and interesting if either of these solutions were stable under a more detailed analysis). As with the domain walls, this indication that the phantom ball solutions are unstable or unphysical is good from the physical standpoint in that both of these solutions would not remain around long due to their postulated instability. However, these two solutions might form in the early Universe and leave some signature on observables from the early Universe (e.g., they might leave an small imprint on the $\mathrm{CMB}$ ) yet due to their postulated instability, decay away before causing problems with cosmological observations like those associated with regular domain walls and/or GUT magnetic monopoles.
In Sec. V, we found wormhole solutions supported by the phantom fields. The wormhole solutions were the only solutions of the four types that we studied that had (at least for some set of parameters) positive mass as shown by Fig. 19. Since these solutions did not have any horizons (i.e., the metric ansatz functions, $A(r)$ and $B(r)$, did not got to zero or become negative), the wormholes are traversable "in principle" meaning [27] simply that they do not have horizons. Determining if these wormhole solutions are traversable "in practice" (meaning that the tidal forces can be engineered to be small enough to allow an observer to pass through the wormhole throat without being tidally disrupted [27]) is difficult, given that the solutions were obtained numerical. However, given the wide range of shapes for the metric ansatz functions $A(r)$ and $B(r)$, it is likely that one could also engineer these phantom wormholes to be traversable "in practice".

Finally, in Sec. VI, we found global, phantom cosmic string solutions. Similar to the cases of the phantom ball solutions and phantom wormhole solutions, the equation of state parameter satisfied $w<-1$ for some range of $\rho$, as shown in Fig. 25, making the term phantom cosmic string appropriate. Also, as in the case of the phantom ball solutions, although for some ranges of $\rho$ the energy density of these cosmic strings was positive, $T_{0}^{0}>0$, the mass per unit length was negative, $\mu_{c s}<0$. (There was a very particular choice of initial conditions, namely, $\phi(0)=0$ and $\chi(0)=0$, which might have given a very small, positive $\mu_{c s}$, but we could not determine if this was a numerical artifact or not). As with the domain wall solutions and the phantom ball solutions, the fact that $\mu_{c s}<0$ indicates that these solutions are likely not stable. Now in the case of the domain wall and phantom ball solutions, this postulated instability is desirable from a physical point of view, since ordinary domain walls and GUT magnetic monopole solutions (the rough equivalent of the phantom ball solutions) cause difficulties in regards to cosmological observations. On the other hand normal cosmic strings are thought to play a potential role in structure formation [7] in the Universe. The postulated instability of our phantom cosmic string solution would rule out their use for structure formation, but as with the domain wall and phantom ball solutions, the phantom cosmic strings might leave some imprint on cosmological observables such as the CMB.

\section{ACKNOWLEDGMENTS}

This work was supported by Grant No. $\Phi .0755$ in fundamental research in natural sciences from the Ministry of Education and Science of Kazakhstan. The work of D. S. is supported by a 2015-2016 Fulbright Scholars Grant to Brazil. D. S. wishes to thank the ICTPSAIFR in São Paulo for its hospitality. 
[1] L. Amendola and S. Tsujikawa, Dark Energy: Theory and Observations (Cambridge University Press, Cambridge, England, 2010).

[2] E. Komatsu et al. (WMAP Collaboration), Astrophys. J. Suppl. Ser. 192, 18 (2011).

[3] M. Sullivan et al. (SNLS Collaboration), Astrophys. J. 737, 102 (2011).

[4] P. O. Mazur and E. Mottola, in Proceedings of the Sixth Workshop on Quantum Field Theory under the Influence of External Conditions, University of Oklahoma, September 15-19, 2003, edited by K. A. Milton (Rinton Press, Princeton, NJ, 2004); I. Dymnikova and E. Galaktionov, Classical Quantum Gravity 22, 2331 (2005); F. S. N. Lobo, ibid. 23, 1525 (2006); A. DeBenedictis, D. Horvat, S. Ilijic, S. Kloster, and K. S. Viswanathan, ibid. 23, 2303 (2006); A. DeBenedictis, R. Garattini, and F. S. N. Lobo, Phys. Rev. D 78, 104003 (2008); V. Gorini, U. Moschella, A. Y. Kamenshchik, V. Pasquier, and A. A. Starobinsky, ibid. 78, 064064 (2008); V. Gorini, A. Y. Kamenshchik, U. Moschella, O. F. Piattella, and A. A. Starobinsky, Phys. Rev. D 80, 104038 (2009).

[5] V. Dzhunushaliev, V. Folomeev, R. Myrzakulov, and D. Singleton, J. High Energy Phys. 07 (2008) 094; K. A. Bronnikov and J. C. Fabris, Phys. Rev. Lett. 96, 251101 (2006); K. A. Bronnikov, H. Dehnen, and V. N. Melnikov, Gen. Relativ. Gravit. 39, 973 (2007).

[6] K. A. Bronnikov, Acta Phys. Pol. B 4, 251 (1973); H. G. Ellis, J. Math. Phys. (N.Y.) 14, 104 (1973); Gen. Relativ. Gravit. 10, 105 (1979); T. Kodama, Phys. Rev. D 18, 3529 (1978); T. Kodama, L. C. S. de Oliveira, and F. C. Santos, Phys. Rev. D 19, 3576 (1979); M. S. Morris and K. S. Thorne, Am. J. Phys. 56, 395 (1988); M. S. Morris, K. S. Thorne, and U. Yurtsever, Phys. Rev. Lett. 61, 1446 (1988); P. K. F. Kuhfittig, Adv. Stud. Theor. Phys. 5, 365 (2011); L. X. Li, J. Geom. Phys. 40, 154 (2001); C. Armendariz-Picon, Phys. Rev. D 65, 104010 (2002); S. V. Sushkov and S. W. Kim, Classical Quantum Gravity 19, 4909 (2002); J. P. S. Lemos, F. S. N. Lobo, and S. Q. de Oliveira, Phys. Rev. D 68, 064004 (2003); F. S. N. Lobo, Phys. Rev. D 71, 084011 (2005); S. V. Sushkov, Phys. Rev. D 71, 043520 (2005); A. Nakonieczna, M. Rogatko, and R. Moderski, Phys. Rev. D 86, 044043 (2012); A. Nakonieczna, M. Rogatko, and Ł. Nakonieczny, J. High Energy Phys. 11 (2015) 012; K. A. Bronnikov and S. V. Sushkov, Classical Quantum Gravity 27, 095022 (2010); K. A. Bronnikov and E. V. Donskoy, Gravitation Cosmol. 17, 176 (2011); K. A. Bronnikov and J. P. S. Lemos, Phys. Rev. D 79, 104019 (2009).

[7] A. Vilenkin and E. P. S. Shellard, Cosmic Strings and Other Topological Defects (Cambridge University Press, Cambridge, England, 2000).

[8] J. Magueijo and R. H. Brandenberger, arXiv:astro-ph/ 0002030.

[9] D. Bazeia, H. Boschi-Filho, and F. A. Brito, J. High Energy Phys. 04 (1999) 028.

[10] C. Santos, Classical Quantum Gravity 18, 1835 (2001).

[11] E. R. Bezerra de Mello, Y. Brihaye, and B. Hartmann, Phys. Rev. D 67, 124008 (2003).
[12] M. Cvetic and and H. H. Soleng, Phys. Rep. 282, 159 (1997).

[13] V. A. Berezin, V. A. Kuzmin, and I. I. Tkachev, Phys. Lett. 120B, 91 (1983); Phys. Rev. D 36, 2919 (1987); D. Garfinkle and C. Vuille, Classical Quantum Gravity 6, 1819 (1989); J. R. Ipser and P. Sikivie, Phys. Rev. D 30, 712 (1984); P. Laguna-Castillo and R. A. Matzner, Phys. Rev. D 34, 2913 (1986).

[14] H. Arodz, Phys. Rev. D 52, 1082 (1995); H. Arodz and A. L. Larsen, Phys. Rev. D 49, 4154 (1994); G. Goetz, J. Math. Phys. 31, 2683 (1990); L. M. Widrow, Phys. Rev. D 40, 1002 (1989).

[15] R. Rajaraman, An Introduction to Solitons and Instantons in Quantum Field Theory (North-Holland, Amsterdam, 1982).

[16] D. Bazeia, M. J. dos Santos, and R. F. Ribeiro, Phys. Lett. A 208, 84 (1995); D. Bazeia, J. R. S. Nascimento, R. F. Ribeiro, and D. Toledo, J. Phys. A 30, 8157 (1997); D. Bazeia and A. R. Gomes, J. High Energy Phys. 05 (2004) 012; S. Y. Vernov, Teor. Mat. Fiz. 155, 47 (2008) [Theor. Math. Phys. 155, 544 (2008)]; I. Y. Aref'eva, N. V. Bulatov, and S. Y. Vernov, Theor. Math. Phys. 163, 788 (2010).

[17] V. Dzhunushaliev, K. Myrzakulov, and R. Myrzakulov, Mod. Phys. Lett. A 22, 273 (2007); V. Dzhunushaliev, V. Folomeev, K. Myrzakulov, and R. Myrzakulov, Mod. Phys. Lett. A 22, 407 (2007).

[18] V. Dzhunushaliev and V. Folomeev, Int. J. Mod. Phys. D 17, 2125 (2008).

[19] V. Dzhunushaliev, V. Folomeev, and A. Urazalina, Int. J. Mod. Phys. D 24, 1550097 (2015).

[20] V. Dzhunushaliev, V. Folomeev, K. Myrzakulov, and R. Myrzakulov, Int. J. Mod. Phys. D 17, 2351 (2008).

[21] V. Folomeev, Int. J. Mod. Phys. D 16, 1845 (2007).

[22] V. Dzhunushaliev, Gravitation Cosmol. 13, 302 (2007); V. Dzhunushaliev, V. Folomeev, D. Singleton, and S. Aguilar-Rudametkin, Phys. Rev. D 77, 044006 (2008); V. Dzhunushaliev, V. Folomeev, K. Myrzakulov, and R. Myrzakulov, Gen. Relativ. Gravit. 41, 131 (2009); V. Dzhunushaliev, V. Folomeev, and M. Minamitsuji, Phys. Rev. D 79, 024001 (2009).

[23] R. R. Caldwell, Phys. Lett. B 545, 23 (2002).

[24] P. Jones, G. Muñoz, M. Ragsdale, and D. Singleton, Am. J. Phys. 76, 73 (2008).

[25] R. Wald, General Relativity (Univeristy of Chicago Press, Chicago, 1984).

[26] G. 't Hooft, Nucl. Phys. B 79, 276 (1974); A. M. Polyakov, Zh. Eksp. Teor. Fiz. Pis'ma. 20, 430 (1974) [JETP Lett. 20, 194 (1974)].

[27] M. Visser, Lorentzian Wormholes: From Einstein to Hawking (Woodbury, New York, 1996).

[28] Ya. B. Zeldovich, I. Y. Kobzarev, and L. B. Okun, Sov. Phys. JETP 40, 1 (1975).

[29] V. Dzhunushaliev, V. Folomeev, R. Myrzakulov, and D. Singleton, Phys. Rev. D 82, 045032 (2010).

[30] J. P. Preskill, Phys. Rev. Lett. 43, 1365 (1979). 